\title{
Perturbations of Regularized Determinants of Operators in a Banach Space
}

\author{
Michael Gil \\ Department of Mathematics, Ben Gurion University of the Negev, P.O. Box 653, 84105 Beer-Sheva, Israel \\ Correspondence should be addressed to Michael Gil; gilmi@bezeqint.net
}

Received 15 August 2012; Accepted 7 November 2012

Academic Editor: Mark J. Friedman

Copyright (C) 2013 Michael Gil. This is an open access article distributed under the Creative Commons Attribution License, which permits unrestricted use, distribution, and reproduction in any medium, provided the original work is properly cited.

Let $X$ be a separable Banach space with the approximation property. For an integer $p \geq 1$, let $\Gamma_{p}$ be a quasinormed ideal of compact operators in $X$ with a quasinorm $N_{\Gamma_{p}}$, such that $\sum_{k=1}^{\infty}\left|\lambda_{k}(A)\right|^{p} \leq a_{p} N_{\Gamma_{p}}^{p}(A)\left(A \in \Gamma_{p}\right)$, where $\lambda_{k}(A)$ are the eigenvalues of $A$ and $a_{p}$ is a constant independent of $A$. We suggest upper and lower bounds for the regularized determinants of operators from $\Gamma_{p}$ as well as bounds for the difference between determinants of two operators. Applications to the $p$-summing operators, Hille-Tamarkin integral operators, Hille-Tamarkin matrices, Schatten-von Neumann operators, and Lorentz operator ideals are discussed.

\section{Statement of the Main Result}

Let $X$ be a separable Banach space with the approximation property and the unit operator $I$. Let $E_{p}(z)$ be the Weierstrass primary factor:

$$
\begin{array}{r}
E_{1}(z)=(1-z), \quad E_{p}(z)=(1-z) \exp \left[\sum_{m=1}^{p-1} \frac{z^{m}}{m}\right] \\
(p=2,3, \ldots ; z \in \mathbb{C}) .
\end{array}
$$

For a Riesz operator $A$ whose eigenvalues counted with their algebraic multiplicities are denoted by $\lambda_{k}(A)(k=1,2, \ldots)$, introduce the $p$-regularized determinant

$$
\operatorname{det}_{p}(I-A)=\prod_{k=1}^{\infty} E_{p}\left(\lambda_{k}(A)\right),
$$

provided

$$
\sum_{k=1}^{\infty}\left|\lambda_{k}(A)\right|^{p}<\infty
$$

The classical theory of regularized determinants for Schattenvon Neumann operators has a long history, which is presented, in particular, in $[1,2]$. König [3] developed the theory of regularized determinants for absolutely $p$-summing operators $(2<p<\infty)$ in a Banach space. In [2, 4], following the classical pattern, regularized determinants are defined for operators of the form $I+A$, in a Banach space where not necessarily $A$ itself but at least some power $A^{m}$ admits a trace. The idea is to replace in all formulas the undefined traces by zero.

Let $S N_{p}(p=1,2, \ldots)$ be the von Neumann-Schatten ideal of compact operators $A$ in a separable Hilbert space $H$ with the finite norm $N_{p}(A)=\left[\operatorname{Trace}\left(A A^{*}\right)^{p / 2}\right]^{1 / p}$, where $A^{*}$ is adjoint to $A$. The following inequalities are well-known:

$$
\begin{gathered}
\left|\operatorname{det}_{p}(I-A)\right| \leq \exp \left[d_{p} N_{p}^{p}(A)\right], \\
\left|\operatorname{det}_{p}(I-A)-\operatorname{det}_{p}(I-B)\right| \\
\leq N_{p}(A-B) \exp \left[d_{p}\left(1+\left[N_{p}(A)+N_{p}(B)\right]\right)^{p}\right]
\end{gathered}
$$

with an unknown constant $d_{k}$, see the books [1, page 1106] and [2, page 194]. In $[5,6]$ these inequalities were slightly improved. In [7] it was proved that one can take $d_{p}=\gamma_{p}$, where

$$
\gamma_{p}:=\frac{p-1}{p} \quad(p \neq 1 ; p \neq 3), \quad \gamma_{1}=\gamma_{3}=1 .
$$

In this paper we investigate a quasinormed ideal $\Gamma_{p}$ of compact operators in $X$ with a quasinorm $N_{\Gamma_{p}}(\cdot)$. That is, 
$N_{\Gamma_{p}}(\cdot)$ satisfies all the usual properties of a norm, with the exception of the triangular inequality, which is replaced by

$$
N_{\Gamma_{p}}(A+B) \leq b\left(\Gamma_{p}\right)\left(N_{\Gamma_{p}}(A)+N_{\Gamma_{p}}(B)\right)
$$

with a constant $b\left(\Gamma_{p}\right)$ independent of $A, B$. Moreover, for an integer $1 \leq p<\infty$ and any $A \in \Gamma_{p}$, the inequality

$$
\sum_{k=1}^{\infty}\left|\lambda_{k}(A)\right|^{p} \leq a_{p} N_{\Gamma_{p}}^{p}(A)
$$

holds, where $a_{p}$ is a constant independent of $A$ (but dependent on $\Gamma_{p}$ ). The aim of this paper is to generalize inequalities (4) to operators from $\Gamma_{p}$. In addition, a lower bound for $\operatorname{det}_{p}(I-A)$ is established.

Now we are in a position to formulate our main result.

Theorem 1. Let $A, B \in \Gamma_{p}$ for an integer $p \geq 1$. Then

$$
\begin{aligned}
& \quad\left|\operatorname{det}_{p}(I-A)\right| \leq e^{a_{p} \gamma_{p} N_{\Gamma_{p}}^{p}(A)} \\
& \left|\operatorname{det}_{p}(I-A)-\operatorname{det}_{p}(I-B)\right| \\
& \leq N_{\Gamma_{p}}(A-B) \\
& \quad \times \exp \left[\gamma_{p} a_{p} b\left(\Gamma_{p}\right)\left(1+\frac{1}{2}\left[N_{\Gamma_{p}}(A-B)+N_{\Gamma_{p}}(A+B)\right]\right)^{p}\right] .
\end{aligned}
$$

This theorem is proved in the next section.

Note that if $N_{\Gamma_{p}}$ is a norm, then $b\left(\Gamma_{p}\right)=1$; if $\Gamma_{p}=S N_{p}$, then $a_{p}=1$.

\section{Proof of Theorem 1}

Let $Y$ be an quasinormed space, that is, it is a linear space with a quasinorm $N(\cdot)=N_{Y}(\cdot)$. Namely,

$$
N(C+\widetilde{C}) \leq c(N(C)+N(\widetilde{C})) \quad(C, \widetilde{C} \in Y)
$$

with a constant $c=c_{Y}$.

Lemma 2. For all $C, \widetilde{C} \in Y$, let $f(C+\lambda \widetilde{C})$ be a scalarvalued entire function of $\lambda \in \mathbb{C}$ and there be a monotone nondecreasing function $G:[0, \infty) \rightarrow[0, \infty)$, such that

$$
|f(C)| \leq G(N(C))
$$

for all $C \in Y$. Then

$$
\begin{aligned}
& |f(C)-f(\widetilde{C})| \\
& \quad \leq N(C-\widetilde{C}) G\left(c\left(1+\frac{1}{2} N(C-\widetilde{C})+\frac{1}{2} N(C+\widetilde{C})\right)\right) .
\end{aligned}
$$

Proof. Put

$$
g_{1}(\lambda)=f\left(\frac{1}{2}(C+\widetilde{C})+\lambda(C-\widetilde{C})\right)
$$

Then $g_{1}(\lambda)$ is an entire function and

$$
f(C)-f(\widetilde{C})=g_{1}\left(\frac{1}{2}\right)-g_{1}\left(-\frac{1}{2}\right)
$$

Thanks to the Cauchy integral,

$$
\begin{aligned}
& g_{1}\left(\frac{1}{2}\right)-g_{1}\left(-\frac{1}{2}\right) \\
& \quad=\frac{1}{2 \pi i} \int_{|z|=1 / 2+r} \frac{g_{1}(z) d z}{(z-1 / 2)(z+1 / 2)} \quad(r>0) .
\end{aligned}
$$

Hence,

$$
\begin{aligned}
\left|g_{1}\left(\frac{1}{2}\right)-g_{1}\left(-\frac{1}{2}\right)\right| & \leq\left(\frac{1}{2}+r\right) \sup _{|z|=1 / 2+r} \frac{\left|g_{1}(z)\right|}{\left|z^{2}-(1 / 4)\right|} \\
& \leq \frac{1}{r} \sup _{|z|=1 / 2+r}\left|g_{1}(z)\right| .
\end{aligned}
$$

In addition, by (11),

$$
\begin{aligned}
\left|g_{1}(z)\right| & =\left|f\left(\frac{1}{2}(C+\widetilde{C})+z(C-\widetilde{C})\right)\right| \\
& \leq G\left(N\left(\frac{1}{2}(C+\widetilde{C})+z(C-\widetilde{C})\right)\right) \\
& \leq G\left(\frac{1}{2} c N(C+\widetilde{C})+\left(\frac{1}{2}+r\right) c N(C-\widetilde{C})\right) \\
& \left(|z|=\frac{1}{2}+r\right) .
\end{aligned}
$$

Therefore according to (15),

$$
\begin{aligned}
|f(C)-f(\widetilde{C})| \\
\quad=\left|g_{1}\left(\frac{1}{2}\right)-g_{1}\left(-\frac{1}{2}\right)\right| \\
\quad \leq \frac{1}{r} G\left(\frac{1}{2} c N(C+\widetilde{C})+\left(\frac{1}{2}+r\right) c N(C-\widetilde{C})\right) .
\end{aligned}
$$

Taking $r=1 / N(C-\widetilde{C})$, we get the required result. $2.3]$.

We need also the following result, proved in [7, Lemma

Lemma 3. For any integer $p \geq 1$ and all $z \in \mathbb{C}$, one has $\left|E_{p}(z)\right| \leq \exp \left[\gamma_{p}|z|^{p}\right]$.

Proof of Theorem 1. By the previous lemma

$$
\begin{aligned}
\left|\operatorname{det}_{p}(I-A)\right| & =\prod_{k=1}^{\infty}\left|E_{p}\left(\lambda_{k}(A)\right)\right| \\
& \leq \prod_{k=1}^{\infty} e^{\gamma_{p}\left|\lambda_{k}(A)\right|^{p}} \\
& =\exp \left[\gamma_{p} \sum_{k=1}^{\infty}\left|\lambda_{k}(A)\right|^{p}\right] .
\end{aligned}
$$

Now (8) follows from the latter inequality and (7).

Moreover, (8) and Lemma 2 imply (9). 


\section{Lower Bounds}

Let $1 \notin \sigma(A)$ and $L$ be $a$ Jordan curve connecting 0 and 1 , lying in the disc $\{z \in \mathbb{C}:|z| \leq 1\}$ and such that

$$
\phi_{A}:=\inf _{s \in L ; k=1,2, \ldots}\left|1-s \lambda_{k}(A)\right|>0 .
$$

Let $l=|L|$ be the length of $L$. For example, if $A$ does not have the eigenvalues on $[1, \infty)$, then one can take $L=[0,1]$. In this case $l=1$ and

$$
\phi_{A}=\inf _{s \in[0,1] ; k=1,2, \ldots}\left|1-s \lambda_{k}(A)\right| .
$$

If the spectral radius $r_{s}(A)$ of $A$ is less than one, then $l=1$, $\phi_{A} \geq 1-r_{s}(A)$.

Theorem 4. Let $A \in \Gamma_{p}(p=1,2, \ldots), 1 \notin \sigma(A)$, and condition (20) hold. Then

$$
\left|\operatorname{det}_{p}(I-A)\right| \geq e^{-\left(l a_{p} N_{\Gamma_{p}}^{p}(A) / \phi_{A}\right)} .
$$

Proof. We have

$$
\operatorname{det}_{p}(I-z A)=\prod_{j=1}^{\infty} E_{p}\left(z \lambda_{j}\right) \quad\left(\lambda_{j}=\lambda_{j}(A)\right) .
$$

Clearly,

$$
\begin{gathered}
\frac{d}{d z} \operatorname{det}_{p}(I-z A)=\sum_{k=1}^{\infty} \frac{d E_{p}\left(z \lambda_{k}\right)}{d z} \prod_{j=1, j \neq k}^{\infty} E_{p}\left(z \lambda_{j}\right), \\
\frac{d E_{p}\left(z \lambda_{j}\right)}{d z}=\left[-\lambda_{j}+\left(1-z \lambda_{j}\right) \sum_{m=0}^{p-2} z^{m} \lambda_{j}^{m+1}\right] \exp \left[\sum_{m=1}^{p} \frac{z^{m} \lambda_{j}^{m}}{m}\right] .
\end{gathered}
$$

But

$$
-\lambda_{j}+\left(1-z \lambda_{j}\right) \sum_{m=0}^{p-2} z^{m} \lambda_{j}^{m+1}=-z^{p-1} \lambda_{j}^{p},
$$

since

$$
\sum_{m=0}^{p-2} z^{m} \lambda_{j}^{m}=\frac{1-\left(z \lambda_{j}\right)^{p-1}}{1-z \lambda_{j}}
$$

So

$$
\begin{aligned}
\frac{d E_{p}\left(z \lambda_{j}\right)}{d z} & =-z^{p-1} \lambda_{j}^{p} \exp \left[\sum_{m=1}^{p} \frac{z^{m} \lambda_{j}^{m}}{m}\right] \\
& =-\frac{z^{p-1} \lambda_{j}^{p}}{1-z \lambda_{j}} E_{p}\left(z \lambda_{j}\right) .
\end{aligned}
$$

Hence,

$$
\frac{d}{d z} \operatorname{det}_{p}(I-z A)=h(z) \operatorname{det}_{p}(I-z A),
$$

where

$$
h(z):=-z^{p-1} \sum_{k=1}^{\infty} \frac{\lambda_{k}^{p}(A)}{1-z \lambda_{k}(A)} .
$$

Consequently,

$$
\operatorname{det}_{p}(I-A)=\exp \left[\int_{L} h(s) d s\right] .
$$

But $|s| \leq 1$ for any $s \in L$, and thus by (7)

$$
\begin{aligned}
\left|\int_{L} h(s) d s\right| & \leq \sum_{k=1}^{\infty}\left|\lambda_{k}(A)\right|^{p} \int_{L} \frac{|s|^{p-1}|d s|}{\left|1-s \lambda_{k}(A)\right|} \\
& \leq \frac{l}{\phi_{A}} \sum_{k=1}^{\infty}\left|\lambda_{k}(A)\right|^{p} \leq \frac{l a_{p}}{\phi_{A}} N_{\Gamma_{p}}^{p}(A) .
\end{aligned}
$$

Therefore,

$$
\begin{aligned}
\left|\operatorname{det}_{p}(I-A)\right| & =\left|\exp \left[\int_{L} h(s) d s\right]\right| \\
& \geq \exp \left[-\left|\int_{L} h(s) d s\right|\right] \\
& \geq \exp \left[-N_{\Gamma_{p}}^{p} l a_{p} \phi_{A}^{-1}(A)\right],
\end{aligned}
$$

Since

$$
\begin{aligned}
& \quad\left|\operatorname{det}_{p}(I-B)\right| \\
& \quad \geq\left|\operatorname{det}_{p}(I-A)\right|-\left|\operatorname{det}_{p}(I-A)-\operatorname{det}_{p}(I-B)\right|,
\end{aligned}
$$

Theorems 1 and 4 imply the following result.

Corollary 5. Let $A, B \in \Gamma_{p}$ for an integer $p \geq 1,1 \notin \sigma(A)$, and condition (20) hold. If, in addition,

$$
\begin{aligned}
& \exp \left[-\frac{a_{p} l N_{\Gamma_{p}}^{p}(A)}{\phi_{A}}\right] \\
& >N_{\Gamma_{p}}(A-B) \\
& \quad \times \exp \left[a_{p} \gamma_{p} b\left(\Gamma_{p}\right)\left(1+\frac{1}{2}\left(N_{\Gamma_{p}}(A+B)+N_{\Gamma_{p}}(A-B)\right)\right)^{p}\right],
\end{aligned}
$$

then $I-B$ is invertible.

\section{Applications}

Suppose $1 \leq p<\infty$ and that $A$ a linear operator in $X$. $A$ is said to be $p$-summing, if there is a constant $v$ such that 
regardless of a natural number $m$ and regardless of the choice $x_{1}, \ldots, x_{m} \in X$ we have

$$
\begin{aligned}
& {\left[\sum_{k=1}^{m}\left\|A x_{k}\right\|^{p}\right]^{1 / p}} \\
& \quad \leq v \sup \left\{\left[\sum_{k=1}^{m}\left|\left(x^{*}, x_{k}\right)\right|^{p}\right]^{1 / p}: x^{*} \in X^{*},\left\|x^{*}\right\|=1\right\},
\end{aligned}
$$

cf. [8]. The least $v$ for which this inequality holds is denoted by $\pi_{p}(A)$. The set of $p$-summing operators in $X$ with the finite norm $\pi_{p}$ is an ideal, cf. [9], which is denoted by $\Pi_{p}$. By the well-known Theorem 3.7.2 in [9, page 159],

$$
\sum_{k=1}^{\infty}\left|\lambda_{k}(A)\right|^{p} \leq \pi_{p}^{p}(A) \quad\left(A \in \Pi_{p} ; 2 \leq p<\infty\right)
$$

(see also Theorem 17.4.3 in [?, page 298]). Since $\pi_{p}(A)$ is a norm, Theorems 1 and 4 imply the following.

Corollary 6. Let $A, B \in \Pi_{p}$ for some integer $p \geq 2$. Then $\left|\operatorname{det}_{p}(I-A)\right| \leq \exp \left[\gamma_{p} \pi_{p}^{p}(A)\right]$ and

$$
\begin{aligned}
& \left|\operatorname{det}_{p}(I-A)-\operatorname{det}_{p}(I-B)\right| \\
& \quad \leq \pi_{p}(A-B) \exp \left[\gamma_{p}\left(1+\frac{1}{2}\left[\pi_{p}(A-B)+\pi_{p}(A+B)\right]\right)^{p}\right] .
\end{aligned}
$$

If, in addition, (20) holds, then

$$
\left|\operatorname{det}_{p}(I-A)\right| \geq e^{-\left(l \pi_{p}^{p}(A) / \phi_{A}\right)} .
$$

Furthermore, let $L_{\mu}^{p}(\Omega)\left(\Omega \subset \mathbb{R}^{n} ; 1<p<\infty\right)$ be the space of scalar functions $f$ defined on $\Omega$ with a finite positive measure $\mu$ and the norm

$$
\|f\|=\left[\int_{\Omega}|f(x)|^{p} d \mu\right]^{1 / p} .
$$

Let $K: L_{\mu}^{p}(\Omega) \rightarrow L_{\mu}^{p}(\Omega)$ be the integral operator

$$
(K f)(t)=\int_{\Omega} k(t, s) f(s) d \mu
$$

whose kernel $k$ defined on $\Omega \times \Omega$ satisfies the condition

$$
\widehat{k}_{p}(K):=\left[\int_{\Omega}\left(\int_{\Omega}|k(t, s)|^{p^{\prime}} d \mu(s)\right)^{p / p^{\prime}} d \mu(t)\right]^{1 / p}<\infty,
$$

where $1 / p+1 / p^{\prime}=1$. Then $K$ is called a $\left(p, p^{\prime}\right)$-HilleTamarkin operator. As it is well known [8, page 43], any $\left(p, p^{\prime}\right)$-Hille-Tamarkin operator $K$ is a $p$-summing operator and

$$
\pi_{p}(K) \leq \widehat{k}_{p}(K) .
$$

Since $\widehat{k}_{p}(\cdot)$ is a norm, by Theorems 1 and 4 we get.
Corollary 7. Let $K$ and $\widetilde{K}$ be ( $\left.p, p^{\prime}\right)$-Hille-Tamarkin operators in $L_{\mu}^{p}(\Omega)$ for an integer $p \geq 2$. Then $\left|\operatorname{det}_{p}(I-K)\right| \leq$ $\exp \left[\gamma_{p} \widehat{k}_{p}^{p}(K)\right]$ and

$$
\begin{aligned}
& \quad\left|\operatorname{det}_{p}(I-K)-\operatorname{det}_{p}(I-\widetilde{K})\right| \\
& \quad \leq \widehat{k}_{p}(\widetilde{K}-K) \exp \left[\gamma_{p}\left(1+\frac{1}{2}\left[\widehat{k}_{p}(\widetilde{K}-K)+\widehat{k}_{p}(\widetilde{K}+K)\right]\right)^{p}\right] .
\end{aligned}
$$

If, in addition, condition (20) holds for $A=K$, then

$$
\left|\operatorname{det}_{p}(I-K)\right| \geq e^{-\left(\widehat{l k}_{p}^{p}(K) / \phi_{K}\right)} .
$$

Now let us consider a linear operator $T$ in $l^{p}(1<p<\infty)$ generated by an infinite matrix $\left(t_{j k}\right)_{j, k=1}^{\infty}$, satisfying

$$
\widehat{\tau}_{p}(T):=\left[\sum_{j=1}^{\infty}\left(\sum_{k=1}^{\infty}\left|t_{j k}\right|^{p^{\prime}}\right)^{p / p^{\prime}}\right]^{1 / p}<\infty .
$$

Then $T$ is called a $\left(p, p^{\prime}\right)$-Hille-Tamarkin matrix. As it is well known [8, page 43], any ( $\left.p, p^{\prime}\right)$-Hille-Tamarkin matrix $T$ is a $p$-summing operator with

$$
\pi_{p}(T) \leq \widehat{\tau}_{p}(T),
$$

cf. [9, Sections 5.3.2 and 5.3.3, page 230].

Since $\widehat{\tau}_{p}(\cdot)$ is a norm, Theorems 1 and 4 imply the following.

Corollary 8. Let $T$ and $\widetilde{T}$ be ( $\left.p, p^{\prime}\right)$-Hille-Tamarkin matrices for an integer $p \geq 2$. Then $\left|\operatorname{det}_{p}(I-T)\right| \leq \exp \left[\gamma_{p} \widehat{\tau}_{p}^{p}(T)\right]$ and

$$
\begin{aligned}
& \left|\operatorname{det}_{p}(I-T)-\operatorname{det}_{p}(I-\widetilde{T})\right| \\
& \leq \widehat{\tau}_{p}(\widetilde{T}-T) \\
& \quad \times \exp \left[\gamma_{p}\left(1+\frac{1}{2}\left[\widehat{\tau}_{p}(\widetilde{T}-T)+\widehat{\tau}_{p}(\widetilde{T}+T)\right]\right)^{p}\right] .
\end{aligned}
$$

If, in addition, condition (20) holds for $A=T$, then

$$
\left|\operatorname{det}_{p}(I-T)\right| \geq e^{-\left(\tau_{p}^{p}(T) / \phi_{T}\right)} .
$$

Now let $X=H$ be a separable Hilbert space and $L_{q, r}(q>$ $1,0<r<q$ ) the Lorentz ideal of compact operators $T$ with the finite quasinorm

$$
N_{q, r}(T)=\left[\sum_{k=1}^{\infty} k^{(q / r)-1} s_{k}^{q}(T)\right]^{1 / q},
$$

where $s_{k}(T)$ are the singular numbers of $T$ taken with their multiplicities. So

$$
\begin{array}{r}
N_{q, r}(T+\widetilde{T}) \leq c_{q, r}\left(N_{q, r}(T)+N_{q, r}(\widetilde{T})\right) \\
\left(c_{q, r}=\text { const; } T, \widetilde{T} \in L_{q, r}\right) .
\end{array}
$$


For the details, see [?, Section 1.1]. By [?, Lemma 1.4],

$$
\sum_{k=1}^{\infty} k^{(q / r)-1}\left|\lambda_{k}(T)\right|^{q} \leq c_{q, r} N_{q, r}^{q}(T) .
$$

For an integer $p \geq 1$, let $q>p$ and $r=q p /(p+q)$. Then simple calculations show that $(q / r)-1=q / p$. By the Hölder inequality, for $d=q / p$, we obtain

$$
\sum_{k=1}^{\infty}\left|\lambda_{k}(T)\right|^{p} \leq \tau(d)\left(\sum_{k=1}^{\infty} k^{d}\left|\lambda_{k}(T)\right|^{p d}\right)^{1 / d}
$$

with

$$
\tau(d)=\left(\sum_{k=1}^{\infty} k^{-d^{\prime}}\right)^{1 / d^{\prime}} \quad\left(\frac{1}{d^{\prime}}+\frac{1}{d}=1\right) .
$$

So we have

$$
\sum_{k=1}^{\infty}\left|\lambda_{k}(T)\right|^{p} \leq \tau(q / p)\left(\sum_{k=1}^{\infty} k^{(q / r)-1}\left|\lambda_{k}(T)\right|^{q}\right)^{p / q} .
$$

Thus (51) implies the following result.

Lemma 9. For an integer $p \geq 1$ and a $q>p$, let $T \in L_{q, r}$ with $r=q p /(p+q)$. Then

$$
\sum_{k=1}^{\infty}\left|\lambda_{k}(T)\right|^{p} \leq c_{q, r} \tau\left(\frac{q}{p}\right) N_{q, r}^{p}(T) .
$$

Now we can directly apply Theorems 1 and 4 .

\section{References}

[1] N. Dunford and J. T. Schwartz, Linear Operators, Part II: Spectral Theory. Selfadjoint Operators in Hilbert Space, Interscience, New York, NY, USA, 1963.

[2] I. Gohberg, S. Goldberg, and N. Krupnik, Traces and Determinants of Linear Operators, Birkhäuser, Basel, Switzerland, 2000.

[3] H. König, "A Fredholm determinant theory for p-summing maps in Banach spaces," Mathematische Annalen, vol. 247, no. 3, pp. 255-274, 1980.

[4] I. Gohberg, S. Goldberg, and N. Krupnik, "Hilbert-Carleman and regularized determinants for linear operators," Integral Equations and Operator Theory, vol. 27, no. 1, pp. 10-47, 1997.

[5] M. I. Gil', "Upper and lower bounds for regularized determinants," Journal of Inequalities in Pure and Applied Mathematics, vol. 9, no. 1, article 2, p. 6, 2008.

[6] M. I. Gil', "Two-sided bounds and perturbation results for regularized determinants of infinite order compact operators," Journal of Mathematical Analysis and Applications, vol. 349, no. 2, pp. 443-451, 2009.

[7] M. I. Gil', "Ideals of compact operators with the Orlicz norms,Annali di Matematica," Annali di Matematica Pura ed Applicata. In press.

[8] J. Diestel, H. Jarchow, and A. Tonge, Absolutely Summing Operators, Cambridge University Press, Cambridge, UK, 1995.

[9] A. Pietsch, Eigenvalues and s-Numbers, Cambridge University Press, Cambridge, UK, 1987. 


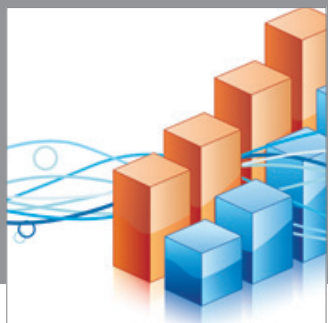

Advances in

Operations Research

mansans

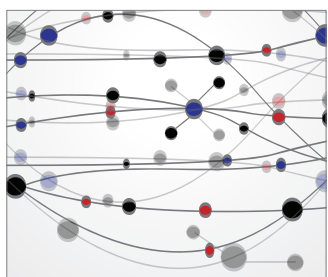

The Scientific World Journal
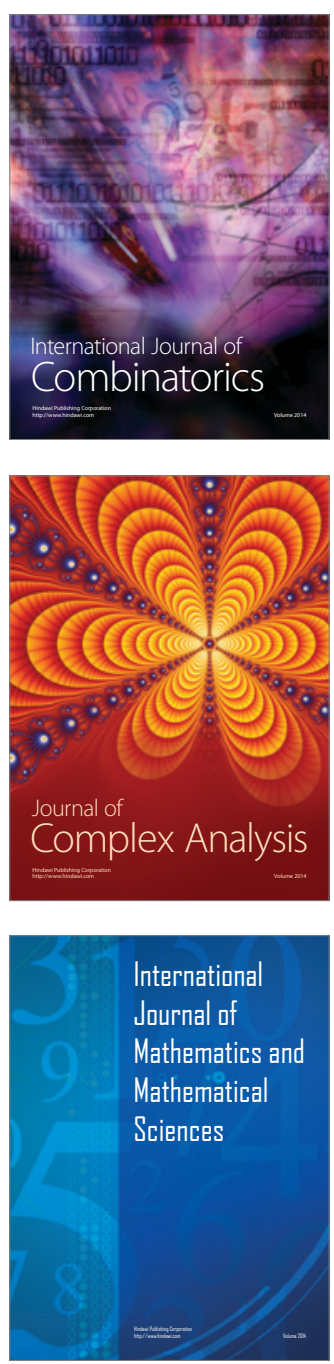
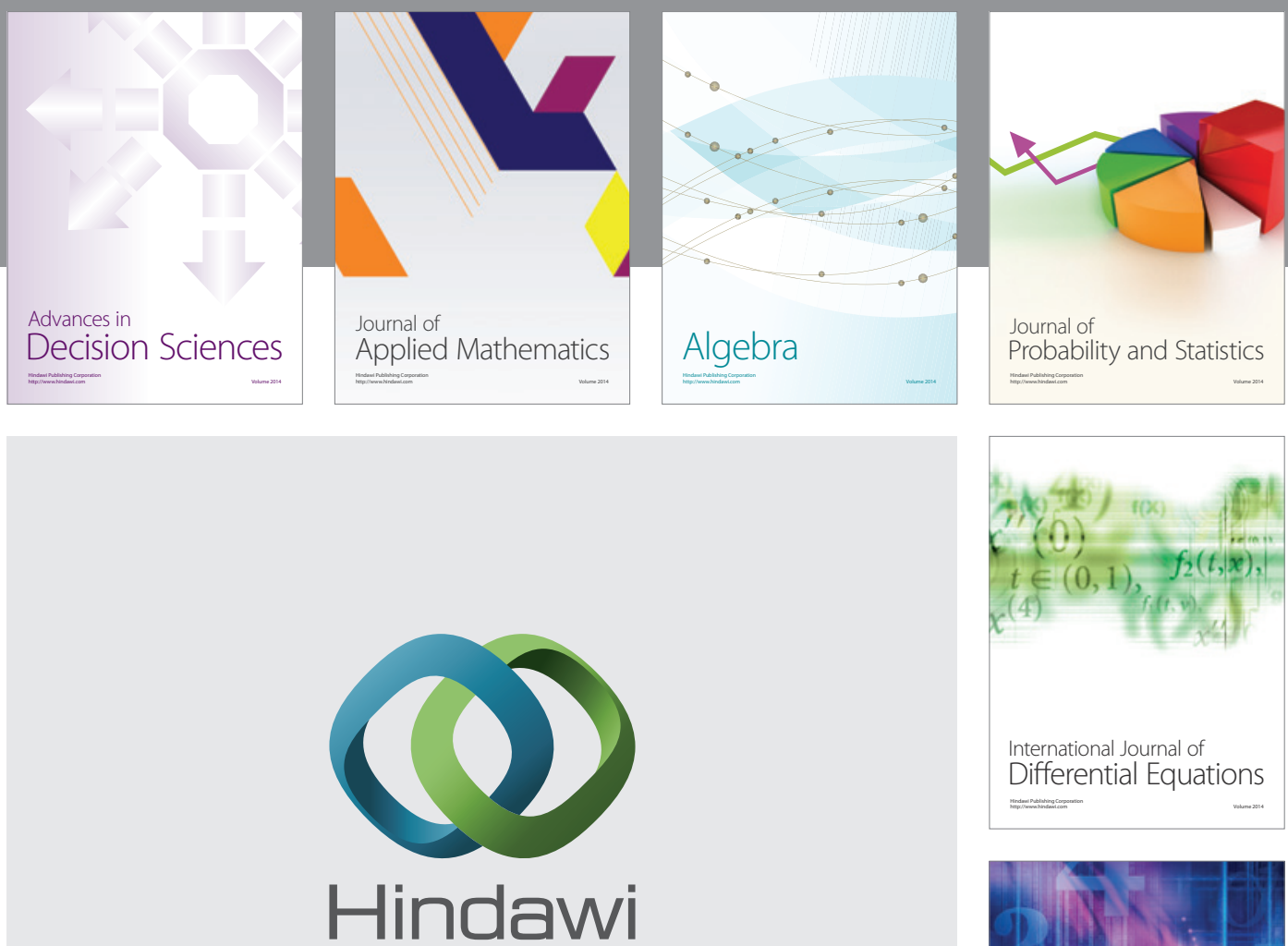

Submit your manuscripts at http://www.hindawi.com
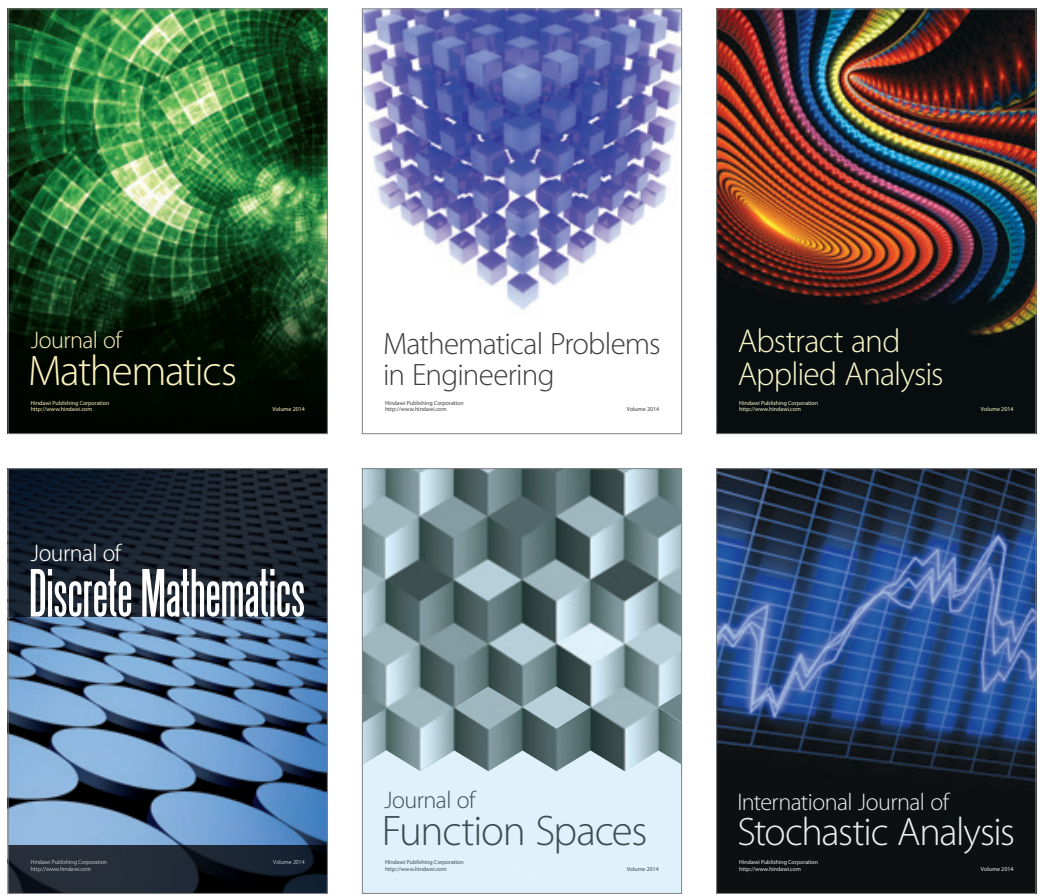

Journal of

Function Spaces

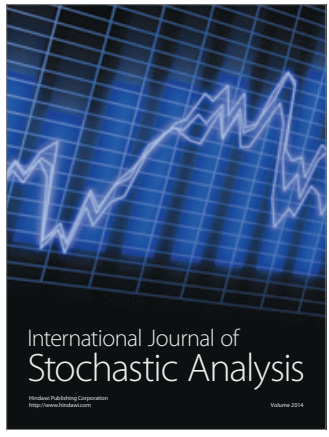

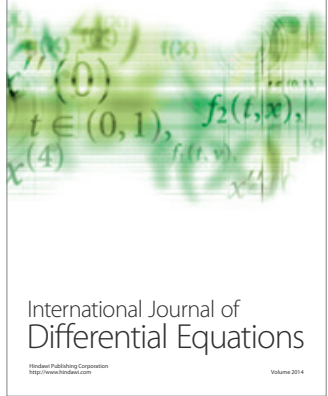
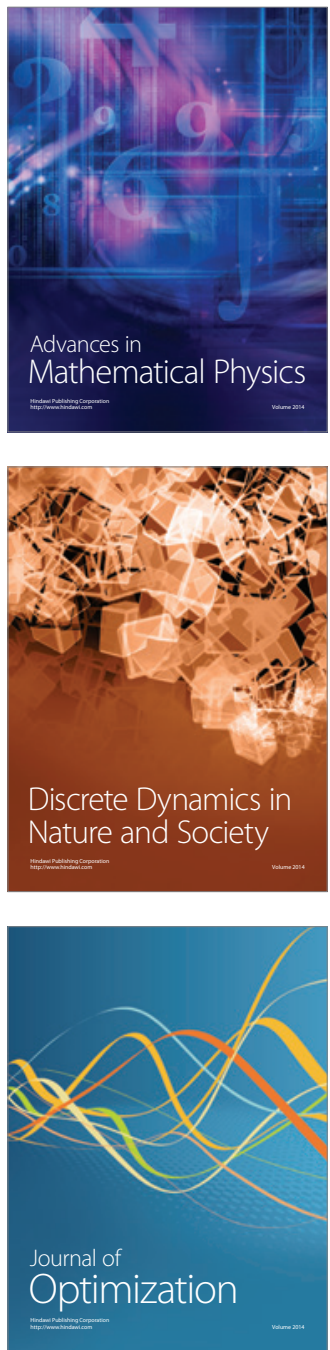